\title{
3D Ultrasound System Using a Magneto-optic Hybrid Tracker for Augmented Reality Visualization in Laparoscopic Liver Surgery
}

\author{
Masahiko Nakamoto ${ }^{1}$, Yoshinobu Sato ${ }^{1}$, Masaki Miyamoto $^{1}$, \\ Yoshikazu Nakamjima ${ }^{1}$, Kozo Konishi ${ }^{2}$, Mitsuo Shimada ${ }^{3}$, \\ Makoto Hashizume $^{2}$, and Shinichi Tamura ${ }^{1}$ \\ 1 Division of Interdisciplinary Image Analysis, \\ Osaka University Graduate School of Medicine \\ 2 Department of Disaster and Emergency Medicine, \\ 3 Department of Surgery II, Graduate School of Medical Science, Kyushu University
}

\begin{abstract}
A three-dimensional ultrasound (3D-US) system suitable for laparoscopic surgery that uses a novel magneto-optic hybrid tracker configuration. Our aim is to integrate 3D-US into a laparoscopic AR system. A 5D miniature magnetic tracker is combined with a $6 \mathrm{D}$ optical tracker outside the body to perform $6 \mathrm{D}$ tracking of a flexible US probe tip in the abdominal cavity. $6 \mathrm{D}$ tracking parameters at the tip are obtained by combining the $5 \mathrm{D}$ parameters at the tip inside the body, the $6 \mathrm{D}$ parameters at the US probe handle outside the body, and the restriction of the tip motion relative to the handle. The system was evaluated in comparison with a conventional 3D ultrasound system. Although the accuracy of the proposed system was somewhat inferior to that of the conventional one, both the accuracy and sweet spot area were found to be acceptable for clinical use.
\end{abstract}

\section{Introduction}

The use of the laparoscope is becoming common as a minimally invasive procedure. However, its restricted views and lack of tactile sensation can limit the surgeon's proficiency as well as make his/her task more stressful. Since laparoscopic surgery is essentially a monitor-based procedure, monitor-based augmented reality (AR) visualization can be naturally integrated into the system so as to both enhance the surgeon's proficiency and reduce stress. For microscopic neurosurgery, which is also naturally combinable with monitor-based AR, AR systems that utilize preoperative CT or MR 3D data have been developed to enhance the surgeon's capability especially in recognizing spatial relationships between tumors and vessels [1] 2. Unlike neurosurgery, in which rigid registration can be assumed, liver surgery requires nonrigid registration so as to be able to register preoperative CT or MR 3D data with the actual liver intraoperatively. However, accurate nonrigid registration between preoperative 3D data and the intraoperative liver is still considered difficult to achieve [3] 4. Currently, ultrasound is regarded as a useful intraoperative imaging modality that allows the surgeon to 
recognize spatial relationships between tumors and vessels of the liver. In this paper, we describe the development of 3D ultrasound (3D-US) for the laparoscope with the aim of integrating 3D-US into an AR system for laparoscopic liver surgery. If we assume that liver motion is negligible between the 3D-US and laparoscopic image acquisitions, nonrigid registration is unnecessary. Such an assumption is clinically valid, since respiratory motion is controllable under anesthesia and breath-holding lasting as long as a minute is attainable without any problem.

3D-US has been used in AR systems, and has been shown to be effective for surgical guidance [5] 6]. Unlike in conventional 3D-US, the tip of a laparoscopecompatible ultrasound probe can be flexibly moved inside the abdominal cavity. However, tracking the probe tip poses a particular challenge. Conventional magnetic trackers are too large to be inserted into the abdominal cavity without an additional incision, while optical trackers suffer from the line of sight constraint. To circumvent these problems, we employ a miniature magnetic tracker only 1 $\mathrm{mm}$ in diameter (Aurora; Northern Digital Inc., Waterloo, OT, Canada), which can be inserted into the abdomen without the need for an additional incision. Although this tracker is suitable for tracking flexible tools inside the body, it has two major restrictions:

- It measures only five degrees of freedom (5D).

- Its sweet spot, within which acceptable accuracy is attainable, is narrow.

To overcome these drawbacks, we employ a novel magneto-optic hybrid configuration [7] in which the reference frames of both the magnetic and optical trackers are registered, with the optical trackers being used to track the mobile field generator of the magnetic tracker. This configuration provides the following advantages:

- Six degrees of freedom (6D) for the position and orientation of the flexible probe tip are measurable by linking it with the optical tracker outside the abdomen.

- Since the laparoscope is rigid, it can be accurately and robustly tracked by the optical tracker outside the abdomen for the superimposition of laparoscopic images and 3D-US.

- The field generator can be arranged so that magnetic tracking is performed within (or near) the sweet spot, which effectively widens the sweet spot of the magnetic tracker.

We previously reported preliminary experimental results 8 for a laparoscopic 3D-US pilot system in which a 5D magnetic tracker was simulated using a conventional 6D magnetic tracker (Fastrak; Polhemus Inc., Colchester, VT) under the simplified assumption that the US probe tip motion relative to the US probe handle outside the abdomen has three degrees of freedom. In this paper, we describe a clinically applicable 3D-US system for laparoscopic AR visualization that utilizes the Aurora miniature magnetic tracker without the above simplified assumption in regard to the probe tip motion. We also evaluate the accuracy of the system. 


\section{Methods}

\subsection{Basic Formulation of Magneto-optic Hybrid Tracker}

A $6 \mathrm{D}$ optical tracker is modeled as a system measuring the transformation $T_{o t \rightarrow o r}$ from the optical tracker frame $\Sigma_{o t}$ to the optical rigid-body frame $\Sigma_{o r}$. A $6 \mathrm{D}$ magnetic tracker is modeled as a system measuring the transformation $T_{m t \rightarrow m r}$ from the magnetic tracker frame $\Sigma_{m t}$ to the magnetic receiver frame $\Sigma_{m r}$. A $6 \mathrm{D}$ magneto-optic hybrid tracker centered at the optical tracker frame $\Sigma_{o t}$ is modeled as a system measuring both the transformation $T_{o t \rightarrow o r}$ the from optical tracker frame $\Sigma_{o t}$, and the transformation $T_{o t \rightarrow m r}$ the from optical tracker frame $\Sigma_{o t}$ to the magnetic receiver frame $\Sigma_{m r}$. A special form of magneto-optic hybrid tracking to measure the transformation $T_{o t \rightarrow m r}$ is formulated as

$$
T_{o t \rightarrow m r}=T_{o t \rightarrow o r} T_{o r \rightarrow m t} T_{m t \rightarrow m r}
$$

where $T_{\text {or } \rightarrow m t}$ represents the transformation from the optical rigid-body frame $\Sigma_{o r}$, which is fixed to the magnetic field generator, to the magnetic tracker frame $\Sigma_{m t}$ 8]. Note that $T_{o r \rightarrow m t}$ is a static transformation while $T_{o t \rightarrow o r}$ and $T_{m t \rightarrow m r}$ change dynamically. If $T_{o r \rightarrow m t}$ is known, the magnetic field generator can be placed anywhere so long as it can be optically tracked. We call the process of obtaining $T_{\text {or } \rightarrow m t}$ "magneto-optic calibration."

\subsection{Basic Formulation of 3D Ultrasound}

Freehand 3D ultrasound using a $6 \mathrm{D}$ tracker is modeled as a system measuring the $3 \mathrm{D}$ coordinates, $\boldsymbol{x}_{s t}$, in some tracker frame from the $2 \mathrm{D}$ coordinates, $\boldsymbol{x}_{u s}$, in a US image frame, which is formulated as

$$
\boldsymbol{x}_{s t}=T_{s t \rightarrow s r} T_{s r \rightarrow u s} \boldsymbol{x}_{u s},
$$

where $T_{s t \rightarrow s r}$ represents the transformation from some tracker frame $\Sigma_{s t}$ to some reference frame $\Sigma_{s r}$, and $T_{s r \rightarrow u s}$ represents the transformation from some reference frame $\Sigma_{s r}$ to a US image frame $\Sigma_{u s}$. $\Sigma_{s t}$ corresponds to $\Sigma_{o t}$ (optical tracker), $\Sigma_{m t}$ (magnetic tracker), and so on. $\Sigma_{s r}$ corresponds to $\Sigma_{o r}$ (optical rigid-body), $\Sigma_{m r}$ (magnetic receiver), and so on. While $T_{s t \rightarrow s r}$ is dynamic, $T_{s r \rightarrow u s}$ is static. The process of obtaining $T_{s r \rightarrow u s}$ is often called " $3 \mathrm{D}$ ultrasound calibration" [6].

\subsection{D Tracking by Combining 5D Magnetic and Additional Optical Trackers}

We consider a 3D-US system described as

$$
\boldsymbol{x}_{o t}=T_{o t \rightarrow p t} T_{p t \rightarrow u s} \boldsymbol{x}_{u s}
$$

where $T_{o t \rightarrow p t}$ represents the transformation from $\Sigma_{o t}$ to the probe tip "moving" frame $\Sigma_{p t}$ (magnetic receiver attached), and $T_{p t \rightarrow u s}$ represents the transformation from $\Sigma_{p t}$ to $\Sigma_{u s}$, which is obtained by preoperative 3D-US calibration. 


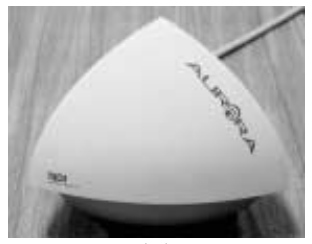

(a)

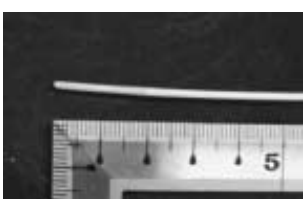

(b)

Fig. 1. Miniature magnetic tracker (Aurora; Northern Digital Inc.). (a) Field generator. (b) Miniature magnetic receiver $1 \mathrm{~mm}$ in diameter.

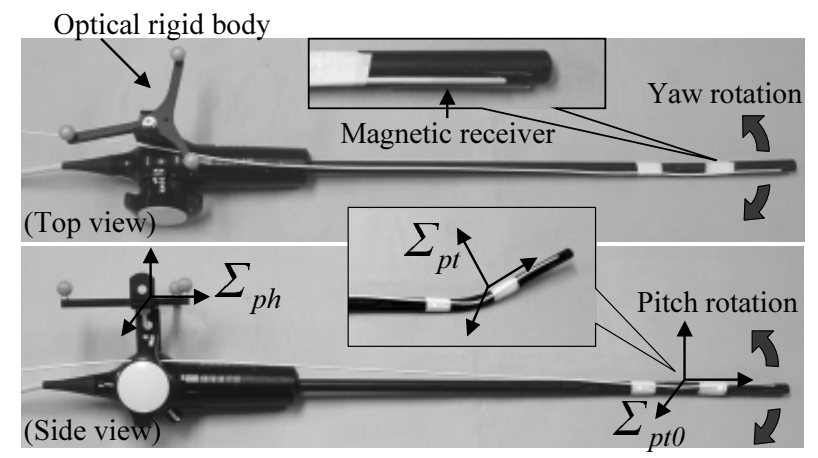

Fig. 2. Ultrasound probe for laparoscope (Aloka, Tokyo).

When the Aurora miniature magnetic tracker (Fig. 1) is used in magneto-optic hybrid tracking, only 5D parameters (2D rotation, 3D translation) are procured in $T_{o t \rightarrow p t}$, the reason being that the $1-\mathrm{mm}$ diameter rod-shaped Aurora magnetic receiver does not provide a rotation parameter around the rod axis. Since 3D parameters are provided with respect to translation, the objective is to obtain the rotation $R_{o t \rightarrow p t}$ using the available $2 \mathrm{D}$ rotational parameters and additional information.

The ultrasound probe for the laparoscope (a 7.5-MHz intraoperative electronic linear probe; Aloka, Tokyo) is shown in Fig. 2 The probe tip inside the abdomen is flexible but its motion is restricted. We define the probe tip "reference" frame $\Sigma_{p t_{0}}$ to describe the restriction of the rotational motion. We describe the probe tip rotation using Pitch-Yaw-Roll angles in $\Sigma_{p t_{0}}$ (Fig. 2.) The main component in the tip rotation is the Pitch component $\theta$, which is controllable using the dial attached to the probe handle located outside the abdomen. The Yaw component $\phi$ can occur due to external force, while the Roll angle can be assumed to be always zero. Thus, the tip rotation $R_{p t_{0} \rightarrow p t}$ is described using Pitch $\theta$ and Yaw $\phi$.

By combining the above rotations, we have

$$
R_{o t \rightarrow p t}=R_{o t \rightarrow p h} R_{p h \rightarrow p t_{0}} R_{p t_{0} \rightarrow p t},
$$

where $R_{o t \rightarrow p h}$ is provided by an optical tracker and $R_{p h \rightarrow p t_{0}}$ is assumed to be known from the preoperative calibration. Since only the $z$-axis direction is provided in $R_{o t \rightarrow p t}$, and $R_{p t_{0} \rightarrow p t}$ can be described by $\theta$ and $\phi$, we have 


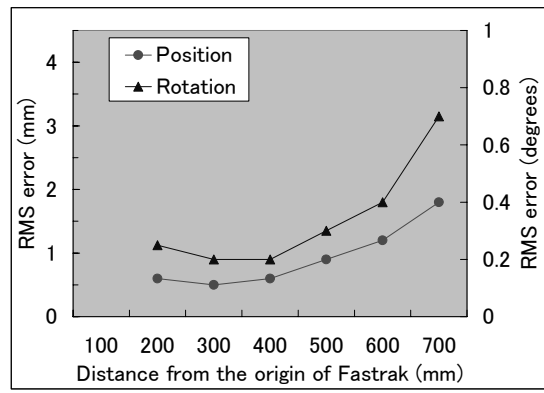

(a) Fastrak.

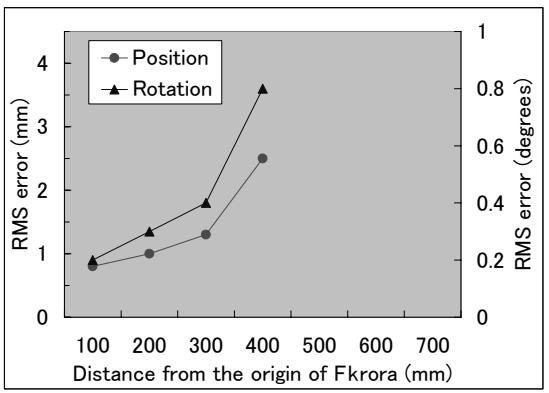

(b) Aurora.

Fig. 3. Positional and rotational errors of magnetic trackers.

$$
\boldsymbol{z}_{o t \rightarrow p t}=R_{o t \rightarrow p h} R_{p h \rightarrow p t_{0}} R_{p t_{0} \rightarrow p t}(\theta, \phi) \boldsymbol{z}_{0},
$$

where $\boldsymbol{z}_{0}=(0,0,1)^{T}$. Solving the above equation, we have $\theta$ and $\phi$, and thus the $6 \mathrm{D}$ parameters of $T_{o t \rightarrow p t}$ are determined.

\subsection{Probe Tip Calibration}

Probe tip calibration is the process of obtaining $T_{p h \rightarrow p t_{0}}$. Firstly, the US probe is put in an arbitrary initial position without external force and $T_{o t \rightarrow p t}$ and $T_{o t \rightarrow p h}$ are obtained. Let $\boldsymbol{t}_{o t \rightarrow p t_{0}}$ and $\boldsymbol{z}_{o t \rightarrow p t_{0}}$ be the translational component and the $z$-axis direction of $T_{o t \rightarrow p t}$ measured at the initial position, respectively. Secondly, the Pitch component $\theta$ is controlled using the dial attached to the probe handle and the $\boldsymbol{t}_{o t \rightarrow p t_{0}}$ positions are obtained at several values of $\theta$. We define the $y$-axis direction, $\boldsymbol{y}_{o t \rightarrow p t_{0}}$, as the normal direction of the plane fitted to these positions. The $x$-axis direction is obtained by $\boldsymbol{x}_{o t \rightarrow p t_{0}}=\boldsymbol{y}_{o t \rightarrow p t_{0}} \times \boldsymbol{z}_{o t \rightarrow p t_{0}}$. Thus, we have

$$
T_{o t \rightarrow p t_{0}}=\left(\begin{array}{cccc}
\boldsymbol{x}_{o t \rightarrow p t_{0}} & \boldsymbol{y}_{o t \rightarrow p t_{0}} & \boldsymbol{z}_{o t \rightarrow p t_{0}} & \boldsymbol{t}_{o t \rightarrow p t_{0}} \\
0 & 0 & 0 & 1
\end{array}\right)
$$

where $\boldsymbol{x}_{o t \rightarrow p t_{0}}, \boldsymbol{y}_{o t \rightarrow p t_{0}}$, and $\boldsymbol{z}_{o t \rightarrow p t_{0}}$ are unit vectors. Finally, by combining $T_{o t \rightarrow p h}$, we have

$$
T_{p h \rightarrow p t_{0}}=T_{o t \rightarrow p h}^{-1} T_{o t \rightarrow p t_{0}} .
$$

\section{Experiments}

Experiments were performed to evaluate the accuracy of the system. Polaris (Northern Digital Inc.) was employed as the optical tracker, and it was combined with either the miniature 5D magnetic tracker, Aurora or the conventional 6D magnetic tracker, Fastrak in order to compare the 3D-US accuracy of these two magneto-optic hybrid systems.

Figure 3 shows the Fastrak and Aurora positional and rotational RMS errors, which arise only from the magnetic tracker itself, measured by a procedure based on the pivot method. The errors were plotted for different distances between the receiver and the origin of each magnetic tracker frame (the field generator). The 


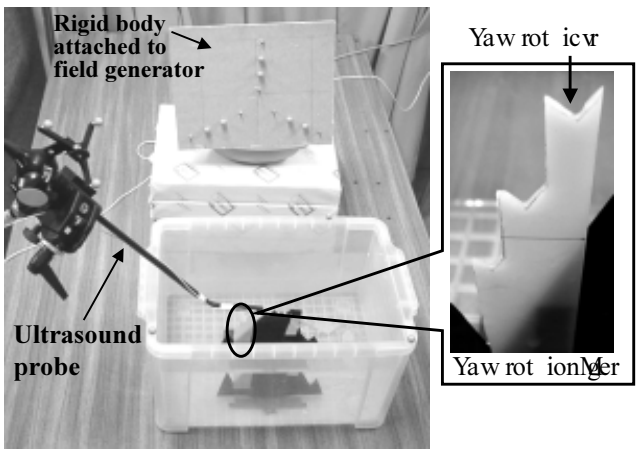

(a)

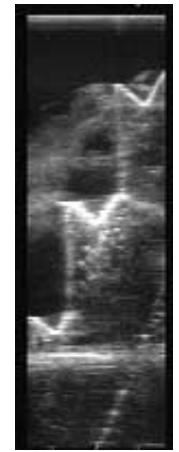

(b)

Fig. 4. Laboratory experiments for accuracy evaluation. (a) Experimental set-up. (b) Ultrasound image of phantom object. Three pit depths are imaged.

accuracy of Aurora was more affected by the distance from the field generator than that of Fastrak 1 . That is, the sweet spot of Aurora was narrower.

Figure 4 shows the experimental set-up (Fig. 4(a) and a US image of a phantom in a water bath (Fig. 4(b)]. The 3D positions at three phantom pit depths in the water bath were measured using the following methods:

1. The Polaris pen-probe digitizer (let $\boldsymbol{x}_{\text {Polaris }}$ be its measurements).

2. The proposed 3D-US system with the Aurora-based hybrid tracker described in 2.3 and 2.4 ( $\left.\boldsymbol{x}_{\text {Aurora }}\right)$.

3. The conventional 3D-US system with the Fastrak-based hybrid tracker $\left(\boldsymbol{x}_{\text {Fastrak }}\right)$, in which the Fastrak 6 D receiver was attached to the probe tip.

As noted earlier, unlike the proposed 3D-US system, the conventional 3D-US system needs an additional incision to be made in the abdomen for its clinical application.

The accuracies of the proposed and conventional 3D-US systems were evaluated by regarding the measurements of the Polaris pen-probe digitizer as the gold standard, that is, the errors were defined as follows:

$$
\begin{aligned}
\Delta \boldsymbol{x}_{\text {Aurora }} & =\left|\boldsymbol{x}_{\text {Polaris }}-\boldsymbol{x}_{\text {Aurora }}\right| \\
\Delta \boldsymbol{x}_{\text {Fastrak }} & =\left|\boldsymbol{x}_{\text {Polaris }}-\boldsymbol{x}_{\text {Fastrak }}\right| .
\end{aligned}
$$

Two sizes of rigid body, each triangular in shape, were used for the optical tracking of the field generator. Polaris markers were attached at the vertices. The larger rigid body has an arc of $180 \mathrm{~mm}$, while that of the smaller one was $120 \mathrm{~mm}$. 3D-US images were acquired for nine different arrangements of field generators of both magnetic trackers relative to the Polaris camera, that is, $\Sigma_{\text {ot }}$. The 2D positions, $\boldsymbol{x}_{u s}$, of the three phantom pits were acquired by manual specification. One hundred measurements for their 3D positions were obtained for each arrangement.

\footnotetext{
${ }^{1}$ Aurora is still under development. Northern Digital Inc. has just announced that the accuracy of Aurora will be improved.
} 


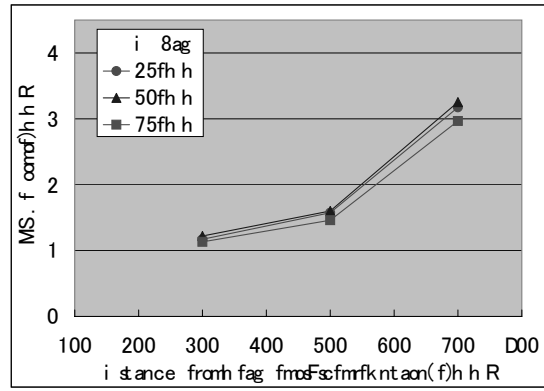

(a) Fastrak.

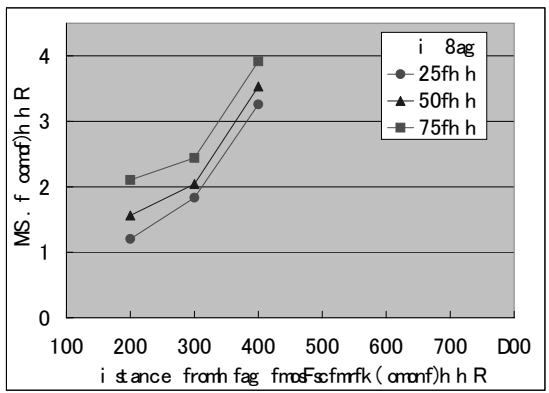

(b) Aurora.

Fig. 5. Positional errors of $3 \mathrm{D}$ ultrasound system. The effects of the distance between the receiver and the origin of each magnetic tracker frame are shown.

Figure 5 shows the RMS errors of the positions estimated by the 3D-US systems for different distances between the receiver and the origin of each magnetic tracker frame. In the conventional (Fastrak-based) system, the error was within $1.5 \mathrm{~mm}$ for all three phantom pit depths when the distance from the tracker origin was within $50 \mathrm{~cm}$. In the proposed (Aurora-based) system, the error was within $2.0 \mathrm{~mm}$ for 50 and $25 \mathrm{~mm}$ depths when the distance from the tracker origin was within $30 \mathrm{~cm}$. In the US images, the effect of depth was thus significant in the proposed method while there was little effect in the conventional system. In both the Fastrak- and Aurora-based systems, the error difference due to the rigid body size for the optical tracking of the field generator was only $0.1-0.2$ $\mathrm{mm}$ between the large and small rigid bodies (results not shown).

\section{Discussion and Conclusions}

We have described a 3D-US system for laparoscopic surgery using a miniature 5D magnetic tracker combined with an optical tracker to realize $6 \mathrm{D}$ tracking inside the abdomen, intended for integration into a laparoscopic AR system. Although the accuracy of the proposed system was somewhat inferior to that of the conventional one, it was acceptable within a $30-\mathrm{cm}$ radius field of view. Since the field generator of the magnetic tracker is mobile inside the field of view of the optical tracker, the sweet spot can be effectively widened. One potential criticism against magneto-optic hybridization is the possibility of error propagation arising from the combining of two trackers. We confirmed both by simulations and laboratory experiments that the increase in error introduced by magneto-optic hybridization was $0.1-0.2 \mathrm{~mm}$ for an appropriate rigid body size as compared that using only a magnetic tracker. Considering that the field generator is mobile, better average accuracy is, in practice, attainable by magneto-optic hybridization.

In our accuracy evaluations, the difference in accuracy between the proposed and conventional 3D-US systems was closely related to the RMS errors of the magnetic trackers themselves. Hence, the accuracy of the proposed system is expected to be improved by developmental improvements in the accuracy of Aurora itself. However, a significant difference was observed in depth dependence in the US images; the dependence was relatively large in the proposed system 
but negligible in the conventional system. This is considered to be due to the inaccuracy of the $1 \mathrm{D}$ rotation parameter, which is not estimated in Aurora, since an error in this rotation results in a proportionate 3D-US depth error. Future work will include analyzing the error factors in the calibration process described in section 2.4 and improvement of the calibration method based on this analysis.

In this paper, we employed two magnetic trackers, Fastrak and Aurora. An alternative choice is miniBird (Ascension Tech Corp., Burlington, VT), a 6D magnetic tracker with a $5 \times 5 \times 10 \mathrm{~mm}^{3}$ receiver. Although the miniBird receiver is smaller than that of Fastrak, in terms of receiver size (as well as cable thickness), Aurora is superior. However, considering the trade-off between size and accuracy, we are planning to evaluate a miniBird-based 3D-US system.

Our proposed 3D-US system has already been integrated into an AR configuration that superimposes 3D-US renderings onto laparoscopic images, and its clinical feasibility in laparoscopic surgery has been tested [9]. The laparoscope, which is a rigid endoscope, is optically tracked, while 3D-US images are obtained using the proposed system. The 3D-US images are superimposed onto those of the laparoscope using the method described in [6]. The AR configuration incorporating the proposed 3D-US system was confirmed to function successfully in the operating room environment 9 .

\section{Acknowledgements}

This work was partly supported by JSPS Research for the Future Program JSPSRFTF99I00903 and JSPS Grant-in-Aid for Scientific Research (B)(2) 12558033.

\section{References}

1. P. J. Edwards, et al. Design and Evaluation of a System for Microscope-Assisted Guided Interventions (MAGI). IEEE Trans. Med. Imaging, 19(11):1082-1093, 2000.

2. Y. Akatsuka, et al. AR Navigation System for Neurosurgery. Lecture Notes in Computer Science, 1935 (MICCAI2000):833-838, 2000.

3. A. J. Herline, et al. Surface Registration for Use in Interactive Image-Guided Liver Surgery. Lecture Notes in Computer Science, 1679 (MICCAI'99):892-899, 1999.

4. Y. Masutani, et al. Modally Controlled Free Form Deformation for Non-rigid Registration in Image-Guided Liver Surgery. Lecture Notes in Computer Science, 2208 (MICCAI2001):1275-1278, 2001.

5. H. Fuchs, et al. Towards Performing Ultrasound-Guided Needle Biopsies from within a Head-Mounted Display. Lecture Notes in Computer Science, 1131 (VBC'96):591$600,1996$.

6. Y. Sato, et al. Image Guidance of Breast Cancer Surgery Using 3-D Ultrasound Images and Augmented Reality Visualization. IEEE Trans. Med. Imaging, 17(5):681693, 1998.

7. M. Nakamoto, et al. Magneto-Optic Hybrid 3-D Sensor for Surgical Navigation. Lecture Notes in Computer Science, 1935 (MICCAI2000):839-848, 2000.

8. Y. Sato, et al. 3D Ultrasound Image Acquisition Using a Magneto-optic Hybrid Sensor for Laparoscopic Surgery. Lecture Notes in Computer Science, 2208 (MICCAI2001):1151-1153, 2001.

9. K. Konishi, et al. Development of AR Navigation System for Laparoscopic Surgery Using Magneto-optic Hybrid Sensor: Experiences with 3 Cases. CARS2002, 2002, in press. 